\title{
A population-based study of the effect of pregnancy history on risk of stillbirth
}

\section{Raisanen, Sari}

2018-01

Raisanen , S , Hogue , C J R , Laine , K , Kramer , M R , Gissler , M \& Heinonen , S 2018 , ' A population-based study of the effect of pregnancy history on risk of stillbirth ' , International Journal of Gynecology \& Obstetrics , vol. 140 , no. 1, pp. 73-80 . https://doi.org/10.1002/ijgo.12342

http://hdl.handle.net/10138/237222

https://doi.org/10.1002/ijgo.12342

unspecified

publishedVersion

Downloaded from Helda, University of Helsinki institutional repository.

This is an electronic reprint of the original article.

This reprint may differ from the original in pagination and typographic detail.

Please cite the original version. 


\title{
A population-based study of the effect of pregnancy history on risk of stillbirth
}

\author{
Sari Räisänen ${ }^{1,2, *, a}$ | Carol J.R. Hogue ${ }^{1}$ | Katariina Laine ${ }^{3}$ | Michael R. Kramer ${ }^{1}$ | \\ Mika Gissler $^{4,5}$ | Seppo Heinonen ${ }^{6}$
}

${ }^{1}$ Department of Epidemiology, Emory University Rollins School of Public Health, Atlanta, GA, USA

${ }^{2}$ Department of Obstetrics and Gynaecology, Kuopio University Hospital, Kuopio, Finland

${ }^{3}$ Department of Obstetrics, Oslo University Hospital, Ullevål, Oslo, Norway

${ }^{4}$ National Institute for Health and Welfare (THL), Helsinki, Finland

${ }^{5}$ Nordic School of Public Health,

Gothenburg, Sweden

${ }^{6}$ Department of Obstetrics and Gynaecology, University of Helsinki and Helsinki University Hospital, Helsinki, Finland

${ }^{*}$ Correspondence

Sari Räisänen, P.O. Box 26, 00014 University of Helsinki, Helsinki, Finland.

Email: shraisanen@gmail.com

${ }^{a}$ Now at the University of Helsinki.

Funding Information

Saastamoinen Foundation; Emil Aaltonen Foundation; Jukes and Uldeen Endowment, Emory University

\begin{abstract}
Objective: To examine the effect of pregnancy history on the risk of stillbirth.

Methods: In a population-based cross-sectional study, data were reviewed from all women aged at least 20 years with singleton pregnancies in Finland between 2000 and 2010. The primary outcome-stillbirth-was defined as fetal death after 22 gestational weeks or death of a fetus weighing at least $500 \mathrm{~g}$.

Results: Among 604047 singleton pregnancies, the prevalence of stillbirth was 3.17 per 1000 deliveries. Prevalence was lowest for multiparous women without previous pregnancy loss after adjusting for major pregnancy complications associated with stillbirth (placenta previa, placental abruption, and pre-eclampsia) and other confounders. Relative to these women, stillbirth prevalence was higher among multiparous women with previous spontaneous abortion and/or stillbirth (adjusted odds ratio [aOR] 1.20, 95\% confidence interval $[\mathrm{Cl}] 1.05-1.36)$, nulliparous women with no previous pregnancy loss (aOR 1.23, 95\% Cl 1.10-1.38), and nulliparous women with prior spontaneous abortion (aOR 1.43, 95\% Cl 1.18-1.74).

Conclusion: Previous pregnancy loss was found to be an independent risk factor for stillbirth, irrespective of the number of prior deliveries.
\end{abstract}

\section{KEYWORDS}

Birth; Childbirth; Population register; Pregnancy history; Pregnancy outcome; Register; Spontaneous abortion; Stillbirth

\section{1 | INTRODUCTION}

Stillbirth is a common adverse perinatal outcome: it complicates approximately 1 of every 200 pregnancies reaching 22 weeks. ${ }^{1}$ Globally, there are an estimated 2.64 million stillbirths every year. ${ }^{2}$ Obstetric complications, placental diseases such as abruption, and genetic or structural abnormalities are reported to be common causes of stillbirth, but the etiology remains unclear in many cases. ${ }^{3,4}$ A recent systematic review and meta-analysis of population-based studies ${ }^{5}$ showed that the major risk factors for stillbirth included nulliparity, obesity (body mass index $\geq 30$ [calculated as weight in kilograms divided by the square of height in meters]), advanced maternal age ( $\geq 35$ years), and smoking during pregnancy. Maternal pre-existing diabetes, ${ }^{5,6}$ pre-eclampsia, ${ }^{5}$ assisted reproductive technology, ${ }^{5,7}$ small-for-gestational-age (SGA) fetus, ${ }^{5}$ SGA in prior pregnancy, ${ }^{8}$ and low education level ${ }^{5}$ have also been shown to be associated with stillbirth.

A recent population-based case-control study in the USA ${ }^{6}$ aimed to identify risk factors for stillbirth that might be identified at the start of pregnancy and reported that pregnancy history was the strongest risk factor for stillbirth (defined as fetal death at $\geq 20$ weeks). Risk of stillbirth was lowest for multiparous women without prior spontaneous abortion or prior stillbirth, and increased progressively for nulliparous women without a previous spontaneous abortion, nulliparous women with history of spontaneous abortion, and then multiparous women with prior stillbirth. ${ }^{6}$ 
In a large population-based study in Sweden, ${ }^{9}$ an increased prevalence of stillbirth was associated with three or more spontaneous abortions among nulliparous women with a singleton pregnancy. A smaller population-based study in Scotland ${ }^{10}$ reported no significant association between prior spontaneous abortion and stillbirth among nulliparous women with singleton pregnancies; however, it reported a twofold higher stillbirth rate among nulliparous women with a prior spontaneous abortion than among women with one prior live birth. Three other studies ${ }^{8,11-13}$ reported a positive association between stillbirth in a first pregnancy and stillbirth in a second pregnancy, but none of the three studies had information on prior spontaneous abortions. It has been suggested that spontaneous abortion and stillbirth have a partly similar pathogenesis, including abnormal placentation. ${ }^{14-17}$ Impaired placentation has also been associated with several major pregnancy complications such as pre-eclampsia, pregnancyinduced hypertension, placental abruption, and SGA-all conditions associated with stillbirth. ${ }^{14,17}$

Finland is a Nordic welfare state with much publicly funded health care, such as free access to prenatal care for all women, and its prevalence of stillbirth is among the lowest in the world. ${ }^{18}$ The aim of the present study was to examine the effect of pregnancy history including information on prior deliveries, prior spontaneous abortion, and prior stillbirth on the risk of stillbirth, taking into account several major pregnancy complications that might cause stillbirth, such as placenta previa, placental abruption, and pre-eclampsia.

\section{2 | MATERIALS AND METHODS}

In a population-based cross-sectional study in Finland, data were reviewed from all women with singleton deliveries between January 1, 2000, and December 31, 2010. The National Institute for Health and Welfare (THL) accepted the study plan and authorized use of the data, as required by the Finnish national data protection legislation (reference no. 1749/5.05.00/2011). Informed consent was not needed.

Information on all live births and stillbirths has been recorded in the Finnish Medical Birth Register (MBR) since 1987. For each delivery, information on sociodemographic characteristics, maternal reproductive history, medical history, pregnancy and delivery characteristics of the index pregnancy, and maternal and neonatal diagnoses during the first postnatal week was gathered. In addition to the MBR, information was obtained from the Population Register Centre on live births and Statistics Finland on stillbirths and deaths during the first week of life. Overall, the data covered $100 \%$ of birth events.

For the present study, MBR data were supplemented by information on maternal medical conditions such as pre-eclampsia, gestational diabetes, and maternal pre-existing diabetes mellitus, on the basis of International Classification of Diseases 10 (ICD-10) codes in the Hospital Discharge Register. This register was established in 1967 and contains information on all diagnoses, medical interventions, and surgical procedures of inpatient and outpatient care in specialized healthcare facilities in Finland. Information on major congenital anomalies defined by ICD-9-CM codes was gathered from the Register of
Congenital Malformations (established in 1963). Encrypted personal identification numbers were used to link the study women's data among the three national health registers, which are all maintained by the THL (register keeper).

For the present analysis, women younger than 20 years were excluded to allow better control of confounding factors. Additionally, multiple births were excluded owing to the higher risk of pregnancy complications. Stillbirth was defined as fetal death after 22 gestational weeks or death of a fetus weighing $500 \mathrm{~g}$ or more. Spontaneous abortion was defined as loss of pregnancy before 22 weeks. Maternal age was classified as 20-29 years, 30-39 years, or 40 years or older. Pregnancy history was classified as one of four groups: nulliparous women (no prior deliveries) without prior spontaneous abortion, nulliparous women with prior spontaneous abortion, multiparous women ( $\geq 1$ prior delivery) without either prior spontaneous abortion or stillbirth, and multiparous women with prior spontaneous and/or stillbirth.

Pregnancy length was estimated on the basis of first- or secondtrimester ultrasonography measurements or from maternal report of the date of her last menstrual period. More than $99 \%$ of pregnant women in Finland have pregnancy length estimated by ultrasonography. In vitro fertilization (IVF) included intracytoplasmic sperm injection and frozen embryo transfer. SGA was identified if the sex- and parity-specific birth weight was more than two standard deviations below the mean, as determined by the Finnish population-based birth curves for 1996-2008. ${ }^{19}$ SGA in a prior pregnancy was defined as birth weight below the 10th percentile. Prior preterm delivery was defined as delivery before 37 weeks. ICD-10 codes were used to identify and define placenta previa (O44), placental abruption (O45), preeclampsia (O14 and O15), gestational diabetes (O24.4), and maternal pre-existing diabetes (O24.0 and O24.1).

Socioeconomic status was defined by maternal occupation at birth and was classified into four groups on the basis of Finland's National Classification on Socioeconomic Position, which follows international recommendations. Socioeconomic status was classified as the following five groups: upper white-collar workers, such as physicians and lawyers; lower white-collar workers, including nurses and secretaries; blue-collar workers, such as cooks and cleaners; others (women with unclassifiable occupations, including entrepreneurs, students, retired, unemployed, and housewives); and missing information. Marital status was classified as in a relationship (married or living with partner), single, or missing information. Smoking habits during pregnancy (reviewed during prenatal visits) were classified as non-smoking, smoking (quit smoking during the first trimester or continued smoking after the first trimester), or missing information.

Major congenital anomalies included structural anomalies, chromosomal defects, and congenital hypothyroidisms. Hereditary diseases and diseases not associated with congenital anomalies, dysfunction of organs or tissues, developmental disabilities, congenital infections, isolated minor dysmorphic features, normal variations and common less significant congenital anomalies were not included, as per the exclusion list of the Register of Congenital Malformations. ${ }^{20}$

The study data were analyzed using SPSS for Windows version 19.0 (IBM, Armonk, NY, USA). Bivariate analyses were performed to 
identify differences between stillbirths and live births using the $\chi^{2}$ test for dichotomous and categorical variables, and Mann-Whitney $U$ test for continuous variables. Missing data were examined for pregnancy history, socioeconomic status, marital status, smoking during pregnancy, and pregnancy history, and multiple data imputation was used.

Unadjusted odds ratios (ORs) and adjusted ORs (aORs) with 95\% confidence intervals (Cls) were estimated by multivariable logistic regression using stillbirth as the dependent variable. Model 1 estimated the unadjusted association between stillbirth (referent: live birth) and pregnancy history (main exposure). Model 2 estimated the association between stillbirth and pregnancy history, adjusting for maternal age, prior SGA, prior preterm delivery, socioeconomic status, smoking, gestational diabetes, pre-existing diabetes, IVF, pre-eclampsia, placenta previa, placental abruption, and major congenital anomalies. Model 3 was a partially adjusted model without major pregnancy complications (placenta previa, placental abruption, and pre-eclampsia) to assess whether such complications mediated an association between stillbirth and reproductive history. As a sensitivity analysis, models 1 and 2 were repeated for the subpopulation of women without prior stillbirth ( $n=3420,0.6 \%)$ and by using non-imputed data. Differences were taken to be significant if $P<0.05$.

\section{3 | RESULTS}

During the study period, there were 640687 deliveries. After women younger than 20 years and multiple births were excluded, data on 604047 deliveries were reviewed. Only 867 (0.1\%) had no information on pregnancy history (Fig. 1).

Overall, there were 3.17 stillbirths per 1000 deliveries (1917/604 047, 0.3\%). Stillbirth was associated with prior SGA, prior preterm delivery, IVF, pre-eclampsia, placenta previa, placental abruption, SGA, and major congenital anomalies (Table 1). Stillbirth was also associated with maternal age, pregnancy history, socioeconomic status, marital status, and smoking during pregnancy (Table 1).

For most variables, the proportion of cases with missing values was very low (Table 1); the only exception was information on socioeconomic status, which had a missing rate of $16.6 \%$ ( $n=100260)$.

The results of unadjusted (model 1) and adjusted (models 2-3) logistic regression analyses using multiple imputed data are presented in Table 2. After adjustment for the known pregnancy complications associated with stillbirth (placenta previa, placental abruption, and pre-eclampsia) and other covariates, pregnancy history was found to be an independent risk factor for stillbirth (model 2). The prevalence of stillbirth was lowest for multiparous women without prior spontaneous abortion and/or prior stillbirth (reference group). Additionally, maternal age seemed to be an independent risk factor for stillbirth: age of 30-39 years and 40 years or more was associated with significantly higher prevalence of stillbirth as compared with 20-29 years. Prior SGA, prior preterm delivery, smoking after first pregnancy trimester, and major congenital anomalies were also independent risk factors for stillbirth. In the partially adjusted model without major pregnancy complications (model 3), the ORs of pregnancy history remained almost unchanged (Table 2 ).

The association between pregnancy history and stillbirth identified in the main analysis (Table 2) was consistent with the findings from a sensitivity analysis restricted to women without prior stillbirth (Table 3) and an analysis using observed rather than imputed data (Table 4).

\section{4 | DISCUSSION}

Identifying which women are at high risk for adverse pregnancy outcomes such as stillbirth, SGA, or severe pre-eclampsia to optimize

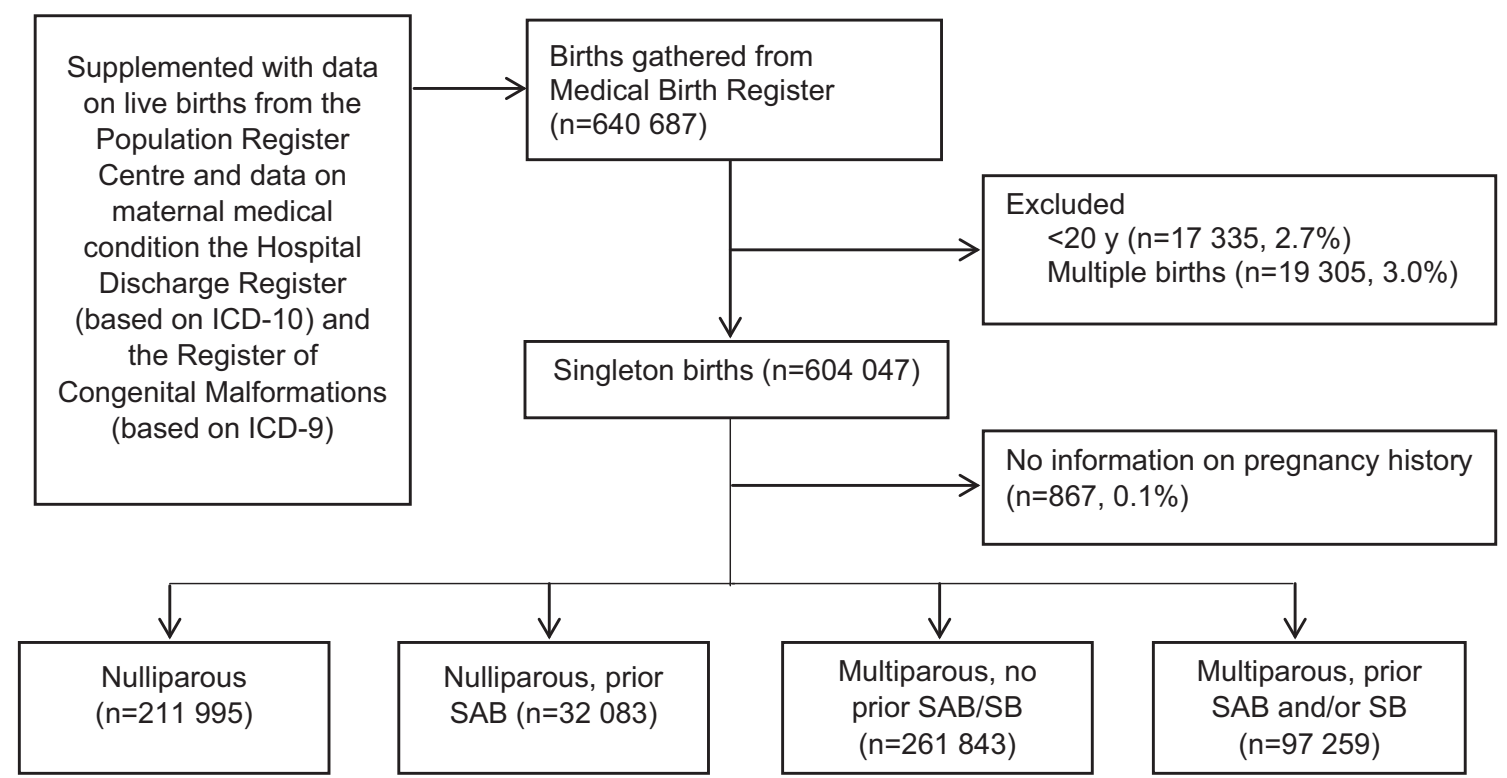

FIGURE 1 Study population. Abbreviations: ICD, International Classification of Diseases; SAB, spontaneous abortion; SB, stillbirth. 
TAB LE 1 Pregnancy and delivery characteristics among women with singleton deliveries (stillbirth and live births) in Finland, 2000-2010. ${ }^{a}$

\begin{tabular}{|c|c|c|c|}
\hline Characteristic & Stillbirth $(n=1917)$ & Live birth $(n=602130)$ & $P$ value $^{b}$ \\
\hline $20-29$ & $837(43.7)$ & $298605(49.6)$ & \\
\hline $30-39$ & $960(50.1)$ & $281463(46.7)$ & \\
\hline$\geq 40$ & $120(6.3)$ & $22062(3.7)$ & \\
\hline Pregnancy history & & & $<0.001$ \\
\hline Multipara, prior spontaneous abortion and/or stillbirth & $371(19.4)$ & $96888(16.1)$ & \\
\hline Nullipara, no prior spontaneous abortion & $664(34.6)$ & $211331(35.1)$ & \\
\hline Nullipara, prior spontaneous abortion & $125(6.6)$ & $31958(5.3)$ & \\
\hline Missing information & $29(1.5)$ & $838(0.1)$ & \\
\hline Upper white-collar worker & $109(5.7)$ & $51556(8.6)$ & \\
\hline Lower white-collar worker & $514(26.8)$ & $213390(35.4)$ & \\
\hline Blue-collar worker & $236(12.3)$ & $88245(14.7)$ & \\
\hline Other $^{\mathrm{c}}$ & $354(18.5)$ & $149383(24.8)$ & \\
\hline Missing information & 704 (36.7) & 99556 (16.5) & \\
\hline Marital status & & & $<0.001$ \\
\hline Married or cohabiting & $1496(78.0)$ & $539417(89.6)$ & \\
\hline Not married or cohabiting & $125(6.5)$ & $35536(5.9)$ & \\
\hline Missing information & $296(15.4)$ & $27177(4.5)$ & \\
\hline In vitro fertilization & $40(2.1)$ & $8728(1.4)$ & 0.020 \\
\hline Pre-eclampsia & $58(3.0)$ & $7503(1.2)$ & $<0.001$ \\
\hline Pre-existing diabetes & $111(5.8)$ & $49086(8.2)$ & $<0.001$ \\
\hline Placenta previa & $15(0.8)$ & $1616(0.3)$ & $<0.001$ \\
\hline Placental abruption & $133(6.9)$ & $1990(0.3)$ & $<0.001$ \\
\hline Pregnancy length, wk & $32.5(6.3)$ & $39.8(1.7)$ & $<0.001$ \\
\hline SGA & $524(30.6)$ & $21287(3.5)$ & $<0.001$ \\
\hline Major congenital anomaly ${ }^{d}$ & 371 (19.4) & 22918 (3.8) & $<0.001$ \\
\hline
\end{tabular}

Abbreviations: SGA, small for gestational age (<2 SD mean birth weight); ICD, International Classification of Diseases.

${ }^{a}$ Values are given as mean \pm SD or number (percentage), unless indicated otherwise.

${ }^{\mathrm{b}} \mathrm{By} \chi^{2}$ or Mann-Whitney $U$ test.

'Included entrepreneurs, students, retired women, unemployed women, housewives, and women with unclassifiable occupations.

IIncluded all structural anomalies, chromosomal defects, and congenital hypothyroidisms identified by ICD-10 codes. Cases of hereditary and other diseases not associated with congenital malformations, dysfunction of organs or tissues, developmental disabilities, congenital infections, isolated minor dysmorphic features, normal variations, and common less significant congenital malformations were not included.

prenatal health care is a challenge in obstetrics. The present study found that prior spontaneous abortion assessed as part of pregnancy history is an independent risk factor for stillbirth even after adjustment for maternal age and major pregnancy complications associated with stillbirth.
Previous studies to determine the association between prior spontaneous abortion and stillbirth are scarce and have reported inconsistent findings. For example, a large population-based study in Sweden ${ }^{9}$ reported a positive association between three or more spontaneous 
TABLE 2 ORs for stillbirth among women with singleton deliveries in Finland in 2000-2010 ( $\mathrm{n}=604$ 047). ${ }^{\mathrm{a}}$

\begin{tabular}{|c|c|c|c|}
\hline Characteristic & Model 1, unadjusted OR (95\% Cl) & Model 2, aOR $(95 \% \mathrm{Cl})$ & Model 3, aOR (95\% Cl) \\
\hline $20-29$ & 1 & 1 & 1 \\
\hline$\geq 40$ & $1.94(1.60-2.35)$ & $1.86(1.52-2.27)$ & $1.96(1.60-2.39)$ \\
\hline \multicolumn{4}{|l|}{ Pregnancy history } \\
\hline Multipara, prior spontaneous abortion and/or stillbirth & $1.37(1.20-1.55)$ & $1.20(1.05-1.36)$ & $1.23(1.08-1.40)$ \\
\hline Nullipara, no prior spontaneous abortion & $1.18(1.01-1.24)$ & $1.23(1.10-1.38)$ & $1.25(1.12-1.40)$ \\
\hline Nullipara, prior spontaneous abortion & $1.38(1.14-1.67)$ & $1.43(1.18-1.74)$ & $1.47(1.21-1.78)$ \\
\hline Prior SGA & $1.89(1.55-2.29)$ & $1.52(1.23-1.87)$ & $1.62(1.32-1.98)$ \\
\hline Lower white-collar worker & $1.08(0.87-1.34)$ & - & - \\
\hline Blue-collar worker & $1.15(0.93-1.43)$ & - & - \\
\hline Other $^{b}$ & $1.06(0.89-1.26)$ & - & - \\
\hline Not married or cohabiting & $1.29(1.10-1.53)$ & - & - \\
\hline \multicolumn{4}{|l|}{ Smoking status } \\
\hline Non-smoking & 1 & 1 & 1 \\
\hline Quit smoking in 1st trimester & $1.00(0.76-1.32)$ & $1.04(0.78-1.37)$ & $1.00(0.76-1.33)$ \\
\hline Smoking after 1st trimester & $1.44(1.26-1.64)$ & $1.47(1.27-1.70)$ & $1.52(1.32-1.76)$ \\
\hline Gestational diabetes & $0.61(0.52-0.73)$ & $0.54(0.41-0.72)$ & $0.53(0.40-0.70)$ \\
\hline
\end{tabular}

Abbreviations: OR, odds ratio; $\mathrm{Cl}$, confidence interval; aOR, adjusted odds ratio; SGA, small for gestational age (<2 SD mean birth weight); ICD, International Classification of Diseases.

aLogistic regression analyses using multiple imputed data. Model 1 was unadjusted. Model 2 was adjusted by maternal age, pregnancy history, prior SGA, prior preterm delivery, smoking status, gestational diabetes, pre-existing diabetes, in vitro fertilization, pre-eclampsia, placenta previa, placental abruption, and major congenital anomaly. Model 3 was adjusted by maternal age, pregnancy history, prior SGA, prior preterm delivery, smoking status, gestational diabetes, pre-existing diabetes, in vitro fertilization, and major congenital anomaly.

${ }^{b}$ Included entrepreneurs, students, retired women, unemployed women, housewives, and women with unclassifiable occupations.

'Included all structural anomalies, chromosomal defects, and congenital hypothyroidisms identified by ICD-10 codes. Cases with hereditary diseases and other diseases not associated with congenital malformations, dysfunction of organs or tissues, developmental disabilities, congenital infections, isolated minor dysmorphic features, normal variations, and common less significant congenital malformations were not included.

abortions and stillbirth (fetal death at $\geq 28$ weeks) among nulliparous women with singleton pregnancy, whereas a small population-based study in Scotland ${ }^{10}$ found no association between prior spontaneous abortion ( $<24$ weeks) and stillbirth (fetal death at $\geq 24$ weeks) among this group of women.

The present results have confirmed that prior stillbirth is associated with stillbirth in subsequent pregnancies, as reported previously. ${ }^{8,11,12}$ However, a direct comparison with other studies is hampered by differences in study design: in some previous investigations, the effect of prior spontaneous abortion was not studied, or the definitions of spontaneous abortion and stillbirth differed from those in the present study. Furthermore, previous studies lacked information on several important covariates associated with stillbirth, including congenital anomalies $^{8-11}$ and adverse pregnancy outcomes such as prior SGA or preterm delivery. ${ }^{8,10-12}$ The present results can be compared with those of a population-based case-control study conducted by the Stillbirth Collaborative Research Network ${ }^{6}$ in the USA, which assessed risk factors identifiable in early pregnancy. That investigation had findings similar to those of the present study; however, it did not control for major pregnancy complications associated with stillbirth owing to study design. In the present study, the association between pregnancy history and stillbirth remained almost unchanged after controlling for major 
TAB LE 3 ORs for stillbirth among women with singleton deliveries, excluding women with prior stillbirths, in Finland in 2000-2010 $(n=600627)^{a}$

\begin{tabular}{|c|c|c|}
\hline Characteristic & Model 1 , unadjusted OR $(95 \% \mathrm{Cl})$ & Model 2, adjusted OR $(95 \% \mathrm{Cl})$ \\
\hline $20-29$ & 1 & 1 \\
\hline$\geq 40$ & $1.93(1.59-2.35)$ & $1.87(1.53-2.29)$ \\
\hline \multicolumn{3}{|l|}{ Pregnancy history } \\
\hline Multipara, prior spontaneous abortion & $1.30(1.15-1.49)$ & $1.17(1.03-1.33)$ \\
\hline Nullipara, no prior spontaneous abortion & $1.13(1.01-1.25)$ & $1.23(1.10-1.38)$ \\
\hline Nullipara, prior spontaneous abortion & $1.40(1.15-1.69)$ & $1.42(1.16-1.72)$ \\
\hline Prior SGA & $1.79(1.46-2.20)$ & $1.52(1.22-1.89)$ \\
\hline Lower white-collar worker & $1.09(0.90-1.30)$ & - \\
\hline Blue-collar worker & $1.14(0.90-1.46)$ & - \\
\hline Other $^{b}$ & $1.05(0.88-1.25)$ & - \\
\hline Not married or cohabiting & $1.29(1.10-1.53)$ & - \\
\hline \multicolumn{3}{|l|}{ Smoking status } \\
\hline Non-smoking & 1 & 1 \\
\hline Quit smoking in 1st trimester & $0.99(0.75-1.30)$ & $1.05(0.79-1.39)$ \\
\hline Smoking after 1 st trimester & $1.50(1.30-1.73)$ & $1.48(1.28-1.71)$ \\
\hline Gestational diabetes & $0.62(0.52-0.74)$ & $0.54(0.41-0.71)$ \\
\hline
\end{tabular}

Abbreviations: OR, odds ratio; $\mathrm{Cl}$, confidence interval; SGA, small for gestational age (<2 SD mean birth weight); ICD, International Classification of Diseases.

aLogistic regression analyses using imputed data. Model 1 was unadjusted. Model 2 was adjusted by maternal age, pregnancy history, prior SGA, prior preterm delivery, smoking status, gestational diabetes, pre-existing diabetes, in vitro fertilization, pre-eclampsia, placenta previa, placental abruption, and major congenital anomaly.

${ }^{\mathrm{b}}$ Included entrepreneurs, students, retired women, unemployed women, housewives and all women with unclassifiable occupations.

'Included all structural anomalies, chromosomal defects, and congenital hypothyroidisms identified by ICD-10 codes. Cases with hereditary diseases and other diseases not associated with congenital malformations, dysfunction of organs or tissues, developmental disabilities, congenital infections, isolated minor dysmorphic features, normal variations, and common less significant congenital malformations were not included.

pregnancy complications, suggesting that pregnancy complications did not mediate the association between pregnancy history and stillbirth.

The present bivariate analyses showed that women with stillbirth had a higher frequency of SGA ( $>2$ SDs lower than mean birth weight) neonates as compared with women with live birth. This finding is in accordance with previous studies to determine the timing of fetal death before stillbirth delivery. ${ }^{5,21}$ Additionally, the present study found that prior SGA was associated with a 1.5-times higher prevalence of stillbirth as compared with women with no prior SGA, in accordance with a Swedish study. ${ }^{8}$ In the present study, index pregnancy information on SGA-a disorder associated with impaired placentation ${ }^{14,17}$-was not used as a covariate because it is a causal factor in the pathway between impaired placentation and stillbirth. Impaired placentation leads to placental dysfunction and is likely to reduce fetal growth, potentially resulting in a live-born SGA neonate or ultimately to stillbirth.

The present findings concerning risk factors for stillbirth are in accordance with previous studies confirming a positive association between stillbirth and advanced maternal age, ${ }^{5}$ smoking during pregnancy, ${ }^{5,6}$ IVF, ${ }^{7}$ and major congenital anomalies. ${ }^{3,4}$ In the present study, 
TABLE 4 ORs for stillbirth among women with singleton births in Finland in 2000-2010, calculated with observed data (n=604 047 ). ${ }^{a}$

\begin{tabular}{|c|c|c|}
\hline Characteristic & Model 1, unadjusted OR $(95 \% \mathrm{Cl})$ & Model 2, adjusted OR (95\% Cl) \\
\hline $20-29$ & 1 & 1 \\
\hline$\geq 40$ & $1.94(1.60-2.35)$ & $1.82(1.49-2.22)$ \\
\hline \multicolumn{3}{|l|}{ Pregnancy history } \\
\hline Multipara, prior spontaneous abortion and/or stillbirth & $1.37(1.21-1.56)$ & $1.21(1.07-1.38)$ \\
\hline Nullipara, no prior spontaneous abortion & $1.13(1.01-1.25)$ & $1.27(1.14-1.42)$ \\
\hline Nullipara, prior spontaneous abortion & $1.40(1.16-1.70)$ & $1.48(1.22-1.80)$ \\
\hline Missing information & $12.41(8.51-18.10)$ & $4.19(2.81-6.27)$ \\
\hline Upper white-collar worker & 1 & - \\
\hline Lower white-collar worker & $1.14(0.93-1.40)$ & - \\
\hline Blue-collar worker & $1.27(1.01-1.59)$ & - \\
\hline Other $^{b}$ & $1.12(0.90-1.39)$ & - \\
\hline Missing information & $3.35(2.73-4.09)$ & - \\
\hline \multicolumn{3}{|l|}{ Marital status } \\
\hline Married or cohabiting & 1 & - \\
\hline Not married or cohabiting & $1.27(1.06-1.52)$ & - \\
\hline Missing information & $3.93(3.47-4.45)$ & - \\
\hline Pre-existing diabetes & $0.69(0.57-0.84)$ & $1.06(0.78-1.44)$ \\
\hline In vitro fertilization & $1.45(1.06-1.98)$ & $1.28(0.93-1.76)$ \\
\hline Pre-eclampsia & $2.47(1.90-3.21)$ & $1.67(1.27-2.20)$ \\
\hline Placenta previa & $2.93(1.76-4.88)$ & $1.80(1.07-3.04)$ \\
\hline Placental abruption & $22.48(18.75-26.96)$ & $17.24(14.26-20.84)$ \\
\hline Major congenital anomaly ${ }^{c}$ & $6.07(5.41-6.80)$ & $5.75(5.13-6.46)$ \\
\hline
\end{tabular}

Abbreviations: OR, odds ratio; $\mathrm{Cl}$, confidence interval; SGA, small for gestational age (<2 SD mean birth weight); ICD, International Classification of Diseases.

a Unconditional logistic regression analyses using non-imputed data. Model 1 was unadjusted. Model 2 was adjusted by maternal age, pregnancy history, prior SGA, prior preterm delivery, smoking status, gestational diabetes, pre-existing diabetes, in vitro fertilization, pre-eclampsia, placenta previa, placental abruption, and major congenital anomaly.

${ }^{b}$ Included entrepreneurs, students, retired women, unemployed women, housewives and all women with unclassifiable occupations.

'Included all structural anomalies, chromosomal defects, and congenital hypothyroidisms identified by ICD-10 codes. Cases with hereditary diseases and other diseases not associated with congenital malformations, dysfunction of organs or tissues, developmental disabilities, congenital infections, isolated minor dysmorphic features, normal variations, and common less significant congenital malformations were not included.

the association between socioeconomic status and stillbirth was statistically nonsignificant in the multivariate analyses. A few studies have reported disparities in socioeconomic status for stillbirth, ${ }^{5}$ but it is difficult to compare the data owing to differences in the definition. The association between disparities in socioeconomic status and stillbirth has been partly explained by a lack of prenatal care; in Finland, however, all pregnant women have free access to publicly funded health care.

The present results did not confirm the positive association between maternal pre-existing diabetes or gestational diabetes and stillbirth 
reported by several previous studies. ${ }^{5,6,22}$ This discrepancy might be explained by the policies of pre-conception planning for all pregnancies among women with pre-existing diabetes and a detailed surveillance program for women with pre-existing and gestational diabetes.

A strength of the present study was the use of validated health registers ${ }^{23,24}$ covering information on the whole population, thereby minimizing selection and misclassification biases. The information on stillbirth was highly reliable because it was gathered from the MBR and supplemented with data compiled by Statistics Finland on stillbirths and deaths during the first week of life. Furthermore, the data included information on the most important known risk factors for stillbirth except maternal weight (gathered since 2004) and major pregnancy complications.

The study has some limitations. The most important potential source of bias was the proportion of missing information on socioeconomic status, marital status, and smoking during pregnancy. The proportion of cases with missing information was substantially higher among women with stillbirth than among women with live birth; this is understandable, because stillbirth is a sensitive situation in which some of the background information might remain unregistered. To counter this, multiple imputed data were used in the multivariable analyses.

On the basis of the present evidence, the most important reproductive risk factors for stillbirth are previous adverse pregnancy outcomes such as prior stillbirth and prior SGA. The present study also showed that a prior spontaneous abortion is an independent risk factor for stillbirth irrespective of prior childbirths. This information could also be used to select nulliparous women at high risk for close obstetric surveillance.

\section{AUTHOR CONTRIBUTIONS}

All authors designed the study, analyzed the data, and drafted the manuscript.

\section{ACKNOWLEDGMENTS}

Funding was provided by the Saastamoinen Foundation, Emil Aaltonen Foundation, and Jukes and Uldeen Endowment, Emory University. The funding sources had no role in the study design, analyses, interpretation of the data, or decision to submit results.

\section{CONFLICTS OF INTEREST}

The authors have no conflicts of interest.

\section{REFERENCES}

1. Flenady V, Middleton P, Smith GC, et al. Lancet's Stillbirths Series steering committee: Stillbirths: The way forward in high-income countries. Lancet. 2011;377:1703-1717.

2. Cousens $\mathrm{S}$, Blencowe $\mathrm{H}$, Stanton $\mathrm{C}$, et al. National, regional, and worldwide estimates of stillbirth rates in 2009 with trends since 1995: A systematic analysis. Lancet. 2011;377:1319-1330.
3. Stillbirth Collaborative Research Network Writing Group. Causes of death among stillbirths. JAMA. 2011;306:2459-2468.

4. Heinonen S, Kirkinen P. Pregnancy outcome after previous stillbirth resulting from causes other than maternal conditions and fetal abnormalities. Birth. 2000;27:33-37.

5. Flenady V, Koopmans L, Middleton P, et al. Major risk factors for stillbirth in high-income countries: A systematic review and metaanalysis. Lancet. 2011;377:1331-1340.

6. Stillbirth Collaborative Research Network Writing Group. Association between stillbirth and risk factors known at pregnancy confirmation. JAMA. 2011;306:2469-2479.

7. Henningsen AA, Wennerholm UB, Gissler M, et al. Risk of stillbirth and infant deaths after assisted reproductive technology: A Nordic study from the CoNARTaS group. Hum Reprod. 2014;29:1090-1096.

8. Surkan PJ, Stephansson O, Dickman PW, Cnattingius S. Previous preterm and small-for-gestational-age births and the subsequent risk of stillbirth. N Engl J Med. 2004;350:777-785.

9. Gunnarsdottir J, Stephansson O, Cnattingius S, Akerud H, Wikstrom AK. Risk of placental dysfunction disorders after prior miscarriages: A population-based study. Am J Obstet Gynecol. 2014;211:34.e1-34.e8.

10. Bhattacharya S, Townend J, Shetty A, Campbell D, Bhattacharya S. Does miscarriage in an initial pregnancy lead to adverse obstetric and perinatal outcomes in the next continuing pregnancy? BJOG. 2008;115:1623-1629.

11. Bhattacharya S, Prescott GJ, Black M, Shetty A. Recurrence risk of stillbirth in a second pregnancy. BJOG. 2010;117:1243-1247.

12. Sharma PP, Salihu HM, Kirby RS. Stillbirth recurrence in a population of relatively low-risk mothers. Paediatr Perinat Epidemiol. 2007;21(Suppl.1):24-30.

13. Lamont K, Scott NW, Jones GT, Bhattacharya S. Risk of recurrent stillbirth: Systematic review and meta-analysis. BMJ. 2015;350:h3080.

14. Plaisier M. Decidualisation and angiogenesis. Best Pract Res Clin Obstet Gynaecol. 2011;25:259-271.

15. Cartwright JE, Fraser R, Leslie K, Wallace AE, James JL. Remodelling at the maternal-fetal interface: Relevance to human pregnancy disorders. Reproduction. 2010;140:803-813.

16. Trogstad L, Magnus P, Stoltenberg C. Pre-eclampsia: Risk factors and causal models. Best Pract Res Clin Obstet Gynaecol. 2011;25: 329-342.

17. Brosens I, Pijnenborg R, Vercruysse L, Romero R. The, "Great Obstetrical Syndromes" are associated with disorders of deep placentation. Am J Obstet Gynecol. 2011;204:193-201.

18. World Health Organization. Stillbirth rate (per 1000 total births). http://apps.who.int/gho/data/node.imr.WHOSIS_000014?lang=en. Accessed October 4, 2017.

19. Sankilampi U, Hannila ML, Saari A, Gissler M, Dunkel L. New population-based references for birth weight, length, and head circumference in singletons and twins from 23 to 43 gestation weeks. Ann Med. 2013;45:446-454.

20. Ritvanen A, Serkiä S. Congenital anomalies 1993-2009. http://www. julkari.fi/bitstream/handle/10024/79900/Tr08_12.pdf?sequence=1. Accessed September 7, 2017.

21. Bukowski R, Hansen NI, Willinger M, et al. Eunice Kennedy Shriver National Institute of Child Health and Human Development Stillbirth Collaborative Research Network: Fetal growth and risk of stillbirth: A population-based case-control study. PLoS Med. 2014;11:e1001633.

22. Gardosi J, Madurasinghe V, Williams M, Malik A, Francis A. Maternal and fetal risk factors for stillbirth: Population based study. BMJ. 2013;346:f108.

23. Gissler M, Shelley J. Quality of data on subsequent events in a routine Medical Birth Register. Med Inform Internet Med. 2002;27:33-38.

24. Sund R. Quality of the Finnish Hospital Discharge Register: A systematic review. Scand J Public Health. 2012;40:505-515. 